\title{
Analisi del discorso sui media e delle voci sociali: Costruzione linguistica e ideologica dei fenomeni di massa in Brasile
}

\section{Análise do Discurso Midiático e Vozes Sociais: Construção Linguística e Ideológica dos Fenômenos de Massa Brasileiros}

DOI: $10.46814 /$ lajdv2n6-001

Recebimento dos originais: 01/09/2020

Aceitação para publicação: 30/10/2020

\author{
Bruno Gomes Pereira \\ Doutor em Letras (Estudos Linguísticos) \\ Docente do Instituto Federal do Tocantins (IFTO) \\ E-mail: bruno.pereira@ifto.edu.br
}

\section{ESTRATTO}

Questo articolo si propone di discutere di come i media brasiliani, in particolare la televisione, ricombinano i discorsi e, con questo, ricostruiscono le immagini dei personaggi dei media che popolano l'immaginario dei telespettatori in Brasile. La Fondazione Teorica è ospitata in Linguistica Applicata nella sua prospettiva interdisciplinare e discorsiva. La Metodologia consiste in una mappatura bibliografica. Concludiamo che le sfere mediatiche brasiliane sono in grado di costruire veri e propri fenomeni di massa, popolando la memoria affettiva di milioni di persone e, con ciò, fruttando guadagni al mercato pubblicitario.

Parole chiave: discorso, Fenomeni di massa, Linguaggio, Televisione.

\section{RESUMO}

Este artigo tem como objetivo discutir como a mídia brasileira, em especial a televisão, recombina discursos e, com isso, reconstrói imagens de figuras midiáticas que povoam o imaginário dos telespectadores no Brasil. A Fundamentação Teórica está alojada na Linguística Aplicada em sua perspectiva interdisciplinar e discursiva. A Metodologia consiste em um mapeamento bibliográfico. Concluímos que as esferas midiáticas brasileiras são capazes de construir verdadeiros fenômenos de massa, ao povoar a memória afetiva de milhões de pessoas e, com isso, render ganhos ao mercado publicitário.

Palavras-Chave: Discurso, Fenômenos de Massa, Linguagem, Televisão.

\section{INTRODUZIONE}

Nel secolo XXI , sono molte le ricerche che problematizzano le questioni sociali, in cui la sfera linguistica stessa non risponde a tutte le domande. Per questo, si deve capire che la linguistica non è altro che una materializzazione lessicogrammatica, influenzata da forze maggiori che promuovono un certo comportamento sociale. In questo senso, siamo d'accordo con Bauman (2004), quando afferma che viviamo in una modernità liquida che, a sua volta, corrobora una serie di interferenze intersociali che rendono le cose più effimere. 
Sempre secondo Bauman (2008), le effimere tra le relazioni nel secolo presente sono eredità di consumo sfrenato, motivate da questioni neoliberiste che muovono il mercato dei consumi e lo alienano per ottenere qualcosa di cui, necessariamente, non ha bisogno.

$\grave{E}$ in questo senso che abbiamo inserito la televisione come punto di partenza per una commercializzazione sfrenata in una società postmoderna. Considerando la visione sistemica della teoria dell'attore-rete, proposta da Latour (2012), è possibile comprendere che il consumo dilagante di un dato prodotto è direttamente associato ad attori sociali, ampiamente diffusi dai media, i quali, pur essendo persone comuni, godono del prestigio di essere nei media televisivi e, con ciò, la portata della massa è fornita in modo più diretto.

Questo articolo è inserito negli studi applicati del linguaggio a carattere non disciplinare (MOITA LOPES, 2006; MOITA LOPES, 2013; SILVA, 2014; PEREIRA, 2014; PEREIRA, 2016; solo per citarne alcuni), poiché riteniamo che Applied Linguistics non lo faccia deve essere vista come una disciplina in sé, ma come un modo emergente di fare scienza, trattando i fenomeni linguistici anche come meccanismi di rappresentazione delle voci sociali periferiche.

Il nostro obiettivo è problematizzare il modo in cui alcuni personaggi pubblici, noti come celebrità dei media, esercitano un potere persuasivo in grado di portare le masse a consumare oggetti diversi senza necessariamente averne bisogno. Pertanto, elenchiamo alcuni nomi che riteniamo essenziali per una discussione soddisfacente sull'argomento. Chiariamo però che, in nessun punto del testo, ci riferiamo alla persona stessa, un cittadino brasiliano, ma piuttosto alla sua immagine di personaggio mediatico capace di muovere il mercato dei consumi.

La metodologia che utilizziamo è di tipo documentario e di approccio qualitativo. Documentario, perché abbiamo ricercato vari documenti sulle influenze di marketing che queste celebrità hanno in Brasile (cfr. SÁ-SILVA, 2009). Abbiamo già scelto l'approccio qualitativo, perché partiamo dalla sfera dell'interpretabilità dell'immanenza dei dati (ANDRÉ, 2013; BORTONIRICARDO, 2008, LAKATOS, MARONI, 2003).

\section{STUDI DI LINGUE APPLICATE}

La linguistica applicata è un aspetto degli studi linguistici in cui la materializzazione linguistica stessa non è sempre la più importante. In questo caso, questioni di carattere pedagogico, psicologico, sociologico e antropologico diventano la spina dorsale per la ricerca in diversi ambiti sociali. Nella sua ricerca, Pereira (2014) stabilisce una storia, sotto forma di una linea temporale, in grado di spiegare i diversi contorni che Applied Linguistics ha acquisito nel tentativo di adattarsi ai bisogni di una società instabile. 
In una ricerca simile, Silva (2014) torna all'idea di indisciplinarietà, coniata da Moita Lopes (2006), nel senso di trattarla come una sorta di palcoscenico più schietto rispetto all'ambiente in cui viviamo. In questo senso, aggiungo all'idea dell'autore alcuni passaggi che hanno attraversato gli studi applicati. Abbiamo, quindi, disciplinare, interdisciplinare, transdisciplinare e indisciplinare, quest'ultimo è il più coerente con ciò che proponiamo in questo approccio.

Di seguito, illustriamo il profilo disciplinare a cui ci riferiamo:

Figura 1: Approccio Disciplinare

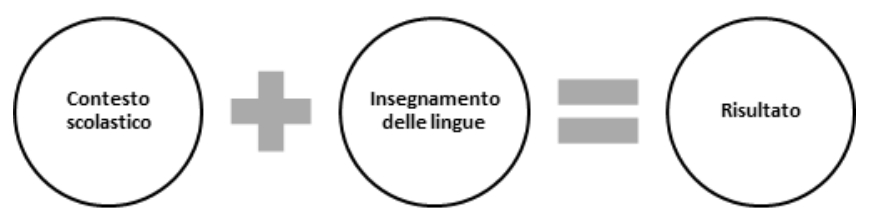

Fonte: Autore

Nella figura sopra, illustriamo la prima concezione di Linguistica Applicata, ancora molto diffusa in Brasile negli anni 80. Essendo questo aspetto nato nel contesto della seconda guerra mondiale, l'intenzione è stata sintetizzata nell'insegnamento delle lingue straniere ai soldati coinvolti.

Il modello dell'immagine illustra l'idea di contesto scolastico, poiché all'epoca era l'unico scopo di questi studi, era proprio quello di concentrarsi sull'ambiente didattico, che, di conseguenza, porta alla seconda sfera, che si occupa dell'insegnamento della lingua. Poiché questo era il principale mezzo di comunicazione tra i soldati, la sua rilevanza era fondamentale. L'insegnamento della lingua era preponderante.

Questa visione della Linguistica Applicata come "teoria" incentrata esclusivamente sull'insegnamento è stata perpetuata da studi linguistici strutturali che, prendendo possesso dell'idea, hanno percepito nella postura disciplinare una sorta di applicazione della linguistica, poiché tutte le teorie proposte da Saussure hanno subito una sorta di riallocazione a insegnamento, dove l'idea di un sistema linguistico era costretto ad adattarsi alle condizioni di insegnamento.

Nel campo della ricerca Linguistica Applicata, la transdisciplinarietà è motivata dalla valorizzazione della conoscenza che va oltre la conoscenza dell'università e inizia a dare voce agli appelli di voci marginalmente svantaggiate. Ciò rappresenta un enorme progresso per gli studi 
linguistici, poiché per comprendere i fenomeni linguistici è necessario comprendere il contesto di vita dei soggetti di ricerca, nonché il rapporto di potere che nasce da questo ascolto delle voci.

$\grave{E}$ in questo senso che, prendendo a prestito le parole di Bourdieu (1989), è necessario percepire il potere simbolico che circonda queste relazioni, spesso implicitamente stabilite. Per il sociologo francese, il tentativo di affrontare forze diverse conferisce potere ai gruppi sociali rimasti indietro. Questa concezione è molto vicina alla nozione di indisciplinarietà, menzionata in precedenza.

Nell'approccio indisciplinare, i movimenti interrelazionali sono più evidenti, poiché l'idea di interruzione disciplinare e l'attenzione data ad altre manifestazioni investigative sono molto più toccanti. In questo senso, correnti di studi differenziati come la pragmatica, la sociologia, la psicologia e altri sono rilevanti per i movimenti indisciplinari che proponiamo.

Pertanto, citiamo i lavori di Pereira (2016), Melo (2015), Silva (2014), Rajagopalan (2014), Moita Lopes (2013; 2006), Kleiman (2013), Fabrício (2006), solo per citarne alcuni.

Pereira (2016) analizza come la scrittura accademica convenzionale differisca dalla scrittura accademica riflessiva, avendo sia manifestazioni ideologiche che discorsive che riposizionano la conoscenza e rassegnano le voci sociali lasciate ai margini. In questo ambiente, consideriamo tali manifestazioni ideologiche come conseguenze semantico-discorsive che aiutano a costruire l'immagine di un mito irraggiungibile nella mente dele.

Seguendo la stessa linea, Melo (2015) problematizza gli scritti riflessivi per capire come si comporta l'io, la persona di cui si parla, in questo scritto. In questo senso, reindirizzo la mia attenzione per capire come questo stabilisca relazioni significative capaci di ricostruire situazioni persuasive. Secondo Fiorin (2006), il rapporto tra l'io e il tu costruisce un percorso generativo di significato capace di mantenere un rapporto più intimo tra l'artista e il destinatario.

Silva (2014) sostiene che l'atteggiamento indisciplinare è valido dal momento in cui si parte dall'empirismo per capire come l'ambiente sociale collabora per il mantenimento delle relazioni interdiscorsive, spesso in modo conflittuale. Tali conflitti sono permeati dalla conoscenza di diversi aspetti che corroborano un rapporto interpersonale in modo più completo e diretto. Per l'autore, la ripresa di voci sociali svalutate è un modo per dare opportunità a gruppi che fino ad allora non sono stati molto importanti nel mondo accademico.

Tali idee sono chiamate da Moita Lopes (2006), e successivamente da Kleiman (2013), come voci dal sud, che sono sociologicamente di gruppi minoritari, emarginati agli occhi della società. Questo broncio è visto, negli studi sociologici, come una lotta di potere che avviene 
implicitamente, quando alcune di queste voci cercano di sovrapporsi e, con ciò, generano un rapporto di eccesso o familiarità.

Pertanto, l'idea di indisciplinarietà, nei termini di Moita Lopes (2013; 2006), è una prospettiva di complessificazione nell'ambito della ricerca accademica, che cerca di stare al passo con i requisiti del Paradigma Emergente. Sia chiamata da Linguistics of Unlearning (2006) o Transgressive Linguistics (FABRÍCIO, 2006), da altri autori, la nozione di indisciplinarietà appare come qualcosa di trasversale, che porta con sé questioni di relazioni di empowerment e comportamento sociale, che è direttamente collegato al consumismo di cui abbiamo parlato fin dall'inizio.

\section{CREAZIONE E PERPETUAZIONE DEI MITI DELLA TELEVISIONE BRASILIANA}

\subsection{ANA MARIA BRAGA}

Ana Maria Braga Maffeis, meglio conosciuta semplicemente come Ana Maria Braga, è la principale presentatrice dei programmi femminili in Brasile. Quando Ana è stata lanciata come leader di programmi per le donne, Ana ha persino eseguito due programmi in diretta su TV Record, che le avrebbero guadagnato innumerevoli ore di lavoro quotidiano. Una routine estenuante, rispetto agli artisti internazionali.

La conduttrice di Mais Você, un programma che porta avanti da oltre un decennio su Rede Globo de Televisão, è il più grande sponsor di sponsorizzazione tra i programmi mattutini giornalieri di Venus Platinada. Con ciò Ana Maria Braga può fregiarsi del titolo di regina della pubblicità, poiché la sua immagine si consolida con il genere di programma che comanda, che genera una sorta di approssimazione tra lo sponsor e la casalinga, di cui si fida seriamente. Nel consiglio dato da Ana decenni fa.

Insieme ad altre grandi star, Ana si distingue per la tranquillità con cui presenta il suo programma, apparendo nella cucina di casa sua. A differenza di altri presentatori dello stesso settore, Ana riesce ad essere popolare, ad innamorarsi della folla, senza essere necessariamente sensazionalista o attraente a causa del maggior numero di spettatori.

\subsection{CHACRINHA}

José Abelardo Barbosa de Medeiros, immortalato come Chacrinha, è stato il più popolare presentatore di programmi di auditorium tra gli anni 1970 e 1980, quando morì. Il suo nome riecheggia, ancora oggi, nel mondo artistico, come sinonimo di talento, disciplina e successo. 
L'influenza di Chacrinha è stata tale che anche le generazioni attuali, che non hanno avuto l'opportunità di vivere i momenti d'oro del "Vecchio Guerriero", come era conosciuto, lo hanno rispettato e rispettato per la storia che ha costruito nella televisione brasiliana. Prova di ciò è stata la sua presenza nella lista delle 100 personalità più importanti nella storia del paese.

Chacrinha è passato da alcune stazioni televisive. Tuttavia, tali cambiamenti non hanno modificato il suo DNA. Il comunicatore ha sempre insistito sul fatto che il ruolo della televisione fosse quello di portare gioia e divertimento. Per questo i suoi programmi erano noti per l'irriverenza con cui trattava i suoi ospiti sul palco. Nel frattempo, ha creato Chacretes, assistenti di scena che sono diventati rapidamente sex symbol e dettavano la moda all'epoca. Rita Cadillac era la più famosa tra loro, portando quell'etichetta fino ad oggi.

La conduttrice in questione aveva una vera sensibilità per scoprire nuovi talenti e godeva anche di un vero tocco di midas. Ogni pubblicista sognava che il proprio prodotto fosse pubblicizzato nel programma Chacrinha e ogni artista si sentiva realizzato solo dopo essere salito sul palco del vecchio guerriero.

Creando slogan, lanciando la moda e acclamando la televisione brasiliana, Chacrinha è diventata una delle figure più iconiche nella storia della televisione in Brasile.

\subsection{FERNANDA MONTENEGRO}

Arlette Pinheiro Esteves Torres, con il nome d'arte di Fernanda Montenegro, è considerata dalla critica e dai media la più grande attrice brasiliana di tutti i tempi. Si dedica all'arte, più precisamente al ruolo di attrice, da oltre mezzo secolo. In quel periodo, ha consolidato una brillante carriera che gli è valsa persino una nomination all'Oscar per la sua interpretazione nel film Central do Brasil.

Con il titolo di "Signora della TV e del teatro", Fernanda Montenegro si è sempre distinta per la sua posizione di artista e cittadina, non esentandosi dal dare un'opinione su alcun argomento. Titolare di una rara eleganza e sensibilità, si consacrò al teatro, al cinema e alla televisione, essendo, in tutti questi veicoli, considerata la migliore.

La memoria affettiva che il Montenegro è riuscito a creare con il suo pubblico è qualcosa di veramente geniale. Qualche volta un'attrice è riuscita ad essere così richiesta per lavori in vari media, facendoli con un'eccellenza naturale.

Nel holl of fame, Dama da Televisão ha accumulato il maggior numero di vittorie nel Troféu Imprensa, un grande riconoscimento per la televisione brasiliana, è stato assegnato il Mário Lago Trophy, un tributo concesso da Rede Globo de Televisão ai più grandi nomi della storia, e inoltre, è 
stata l'unica brasiliana a vincere il premio Emmy International nella categoria Migliore Attrice, per il suo lavoro nella serie Doce de Mãe.

Tra i personaggi più importanti in televisione ci sono Francisca Newman, di Brilhante (1981), Leonarda Furtado, di Cambalacho (1986), Olga Portela, di O Dono do Mundo (1991), Bia Falcão, di Belíssima (2005) e Bete Gouveia, di Passione (2010).

\subsection{HEBE CAMARGO}

Hebe Camargo è il nome del presentatore più prestigioso e famoso della televisione brasiliana. Con il titolo di "Regina della televisione brasiliana", Hebe ha segnato un'epoca e ha fatto piangere il Brasile nel 2012, quando è morto.

Per i critici televisivi, non ci sarà mai un comunicatore come Camargo, che si è distinto per intraprendenza, gioia di vivere, predominio scenico e criticità con cui ha visto la scena politica nazionale. Hebe era consapevole del suo ruolo di opinion maker. Pertanto, non ha esitato a darli, sempre con grande intelligenza e senza paura di quello che poteva accadere.

Proprietaria della propria personalità, Hebe ha contribuito a rivoluzionare la televisione brasiliana dando ai talk show un tocco più moderno in grado di attirare un pubblico sia maschile che femminile. Il comunicatore ha rivoluzionato il segmento in modo così categorico che ha deciso di presentare che i suoi programmi, mostrati il lunedì sera, sono diventati una vera routine nella vita del brasiliano. Tutti hanno dormito dopo la buonanotte della Regina della TV.

Hebe ha il maggior numero di vittorie nel Press Trophy nella categoria Best Presenter, per non parlare del Latin Grammy vinto per aver incoraggiato la cultura brasiliana. Prima di morire, la conduttrice è entrata anche nella lista delle 100 personalità più grandi della storia del Brasile.

\subsection{RENATO ARAGÃO}

Antônio Renato Aragão, o semplicemente Renato Aragão, è uno dei più grandi comici della storia del Brasile. Quando ha guidato il quartetto Os Trapalhões, che ha dato il nome all'omonimo umorista, Renato si è distinto per l'innovazione dell'umorismo nel linguaggio televisivo.

Quando ha optato per un linguaggio più circense, Aragon ha avuto grandi difficoltà nella realizzazione di alcuni progetti all'inizio della sua carriera. Ma quando ha iniziato a contare su altri tre dei suoi colleghi, il Brasile è diventato piccolo per il clamoroso successo.

Durante la proiezione di Os Trapalhões, il Brasile ha smesso di ridere di uno stato d'animo innocente, senza un doppio significato e, soprattutto, sano, come tutta la famiglia potrebbe seguire. 
Aragon e la sua troupe accumulano uno dei più grandi spettatori nelle sale, essendo, fino ad oggi, un riferimento di successo sul grande schermo.

Ma non avendo più lo spazio che merita sulla televisione aperta, Aragon fa parte di un gruppo selezionato di artisti che ha rivoluzionato la televisione e ne ha fatto uno strumento di intrattenimento più leggero e umanizzante.

\subsection{SÍLVIO SANTOS}

Senor Abravanel, meglio conosciuto come Sílvio Santos, è il principale presentatore in Brasile. Ha fatto la storia in televisione e ha fatto una vera fortuna con le vendite e le vendite. La sua eredità è da invidiare, essendo l'artista maschio più ricco nella storia del Brasile.

La principale fonte di reddito è nella sua stazione televisiva Sistema Brasileiro de Televisão, SBT, che spesso disturba la Venere di platino in termini di pubblico.

Il suo programma, mostrato la domenica sera, è quasi sempre al primo posto tra il pubblico della grande São Paulo. Ciò non sarebbe sorprendente se Sílvio Santos non presentasse lo stesso formato di programma da decenni. Nel corso delle generazioni, l'ammirazione del pubblico per "Homem do Baú" non fa che aumentare, rendendolo una delle figure più amate e iconiche della televisione brasiliana.

\subsection{XUXA MENEGHEL}

Maria da Graça Xuxa Meneghel, meglio conosciuta con l'epiteto "Regina dei Bambini”, è la presentatrice brasiliana più popolare da oltre 30 anni. Ha iniziato la sua carriera come modello, all'età di 15 anni, ma è stato come presentatore per bambini del programma Clube da Criança, nel 1983, sull'estinta TV Manchete, che Xuxa sarebbe diventata la più grande star dei programmi per bambini in America Latina.

Il suo passaggio a Rede Globo de Televisão nel 1986 l'ha resa un nome mondiale. Artisti da tutto il pianeta volevano emularlo. Tuttavia, nessuno di loro ha apprezzato il carisma, il talento e il magnetismo di Xuxa. Pertanto, il cosiddetto Regina dei Bambini ha segnato l'epoca del proprio stile di presentazione dei programmi per bambini. Ha sempre trattato il bambino da pari a pari, il che ha suscitato l'interesse dei media e, all'inizio, la stranezza dei genitori, ma mai il ripudio dei bambini.

Il modo specifico in cui Xuxa si è occupata dei bambini l'ha resa la più grande venditrice di dischi nella storia del Brasile e l'unica latina a comparire nelle liste delle 50 donne che hanno venduto di più sul pianeta, occupando il $25^{\circ}$ posto. 
Attraverso un linguaggio giocoso, Xuxa ha fatto festa a milioni di bambini ogni giorno quando è scesa dalla sua nave rosa. I suoi capelli biondi, la bella pelle e gli occhi azzurri l'hanno resa un simbolo di bellezza, che ha suscitato molto l'interesse delle agenzie pubblicitarie. Tutto ciò che porta il suo nome si vende in modo assurdo.

Oggi, su Record TV, nonostante non mostri il risultato atteso all'IBOPE, Xuxa continua a mantenere il titolo di regina, questa volta non solo per i corti, ma per diverse generazioni che la accompagnano fino ad oggi. Il suo programma notturno, il lunedì, è il più grande catalizzatore finanziario di Record, raccogliendo sponsorizzazioni invidiabili per i tempi di crisi.

Per questo motivo, sono numerose le ricerche accademiche che analizzano la potenza di fuoco della regina. Tutte queste ricerche riguardano il potere persuasivo del marchio Xuxa, in campo pedagogico, marketing o consumistico. Tuttavia, è la ricerca di Simpson (1994) che gode di maggiore popolarità quando si tratta della bionda più famosa del Brasile. Nel lavoro, l'autore commenta il successo di Xuxa negli Stati Uniti d'America e lo considera un tipico fenomeno di massa nei paesi in via di sviluppo. Il lavoro presenta una storia della vita di Xuxa e talvolta suggerisce che il comportamento di Xuxa abbia indotto una sessualizzazione precoce e un anti-didatticismo. Tuttavia, Simpson (1994) non si aspettava che un fenomeno di massa così tipico, come ha sottolineato, sarebbe diventato una vera apoteosi senza tempo.

\section{CONSIDERAZIONI FINALI}

Alla luce di quanto sopra, è chiaro che i media sono mezzi di comunicazione estremamente importanti per la divulgazione di una persona cosiddetta "anonima". Il processo di trasformazione dell'anonimato in mito è qualcosa che avviene in modo molto effimero, ma che mobilita contemporaneamente milioni di persone, cambiando radicalmente il loro comportamento.

D'altronde ci sono quei fenomeni di massa che riescono a perpetuarsi nei media e il loro nome non è più solo un semplice soprannome e diventa una sorta di marchio, di entità. Questi casi sono atipici alla luce dell'effimero menzionato da Bauman (2008; 2004) e, quindi, meritano un'attenzione particolare. È necessario capire cosa c'è in queste celebrità che hanno incantato un gran numero di persone per così tanto tempo. Non è nostra intenzione rispondere a questa domanda in modo così diretto, ma piuttosto favorire la riflessione su questo tipo di linguaggio, che, anche se persuasivo e di tendenza, ha caratteristiche senza tempo.

Insomma, i cambiamenti nel comportamento della massa, attraverso l'interferenza discorsiva dei fenomeni di massa, favoriscono un consumo accelerato capace di alterare i più elementari principi di conoscenza e comportamento umano. Pertanto, è necessario ripensare a come queste celebrità 
riescano a reinventarsi nel tempo e continuare a esercitare la stessa influenza negli anni a venire. $\mathrm{Ci}$ auguriamo che questo testo possa contribuire alla ricerca futura, che possa problematizzare con più veemenza questo tipo di comportamento, discorso e ideologia sociale.

\section{RIFERIMENTI}

ANDRÉ, M. O que é um Estudo de Caso Qualitativo em Educação?. Revista da FAEEBA:

Educação e contemporaneidade, Salvador, n. 40, v. 22, p. 95-103, jul./dez. 2013.

BAUMAN, Z. Amor Líquido: sobre a fragilidade dos laços humanos. Rio de Janeiro: Ed. Jorge Zahar, 2004.

BAUMAN, Z. Vida para o Consumo: a transformação das pessoas em mercadorias. Rio de Janeiro: Ed. Jorge Zahar, 2008.

BORTONI-RICARDO, S. M. O professor pesquisador: Introdução à pesquisa qualitativa. São Paulo: Parábola Editorial, 2008.

BOURDIEU, P. O Poder Simbólico. Lisboa: Editora Bertrand Brasil, 1989.

FIORIN, J. L. Introdução ao Pensamento de Bakhtin. São Paulo/SP: Ática, 2006.

KLEIMAN, A. B. Agenda de Pesquisa e Ação em Linguística Aplicada: Problematizações. In.: MOITA LOPES, L. P. (orgs). Linguística Aplicada na Modernidade Recente: Festschrift para Antonieta Celani. São Paulo/SP: Parábola, 2013. p. 39-58.

LAKATOS, E. M.; MARCONI, M. A. Fundamentos da Metodologia Científica. $5^{\text {a }}$ ed. São Paulo: Atlas, 2003.

LATOUR, B. Reagregando o Social: Uma introdução à teoria do Ator-Rede. Salvador/BA: EDUSC, 2012.

MELO, L. C. de. Formas Linguísticas de Inscrição do Outro e do Eu-Mesmo na Escrita Reflexiva Acadêmico-Profissional de Relatórios de Estágio de Professores de Língua. 2015. 167f. Tese (Doutorado em Ensino de Língua e Literatura) - Universidade Federal do Tocantins, Araguaína, 2015.

MOITA LOPES, L. P. da. Da Aplicação de Linguística à Linguística Aplicada Indisciplinar. In.: PEREIRA, R. C.; ROCA, P. (orgs). Linguística Aplicada: Um caminho com diferentes acessos. $1^{\mathrm{a}}$ ed. São Paulo: Contexto, 2013. p. 11-24.

MOITA LOPES, L. P. da. Linguística Aplicada e Vida Contemporânea: Problematização dos construtos que têm orientado a pesquisa. In.: MOITA LOPES, L. P. da (org). Por uma Linguística Aplicada Indisciplinar. São Paulo: Parábola Editorial, 2006. p. 85-108. 
PEREIRA, B. G. Linguística Aplicada no Brasil: um Percurso em Constante Construção. In.: FOSSILE [et al] (orgs). Pesquisas Linguísticas e Demandas do Ensino Básico. Araguaína: Universidade Federal do Tocantins, 2014. p. 189-202 [Livro Eletrônico].

PEREIRA, B. G. Relocalização de Saberes Acadêmicos na Construção de Vozes de Professores em Formação Inicial na Escrita Acadêmica Convencional e Reflexiva. 2016. 350 f. Tese (Doutorado em Ensino de Língua e Literatura) - Universidade Federal do Tocantins, Araguaína, 2016.

SÁ-SILVA, J. R.; ALMEIDA, C. D.; GUINDANI, J. F. Pesquisa Documental: Pistas teóricas e metodológicas. In.: Revista Brasileira de História \& Ciências Sociais, Ano I, n. I. 2009.

SIGNORINI, I.. Do Residual ao Múltiplo e ao Complexo: O objeto da pesquisa em Linguística Aplicada. In.: SIGNORINI, I.; CAVALCANTI, M. C. (orgs). Linguística Aplicada e Transdisciplinaridade. Campinas/SP: Mercado de Letras, 1998. p. 99-110.

SILVA, W. R. Reflexão pela Escrita no Estágio Supervisionado da Licenciatura: Pesquisa em Linguística Aplicada. Campinas: Pontes Editores, 2014.

SIMPSON, A. Xuxa: Megamarketing do sexo, da raça e da modernidade. São Paulo: Editora Sumaré, 1994 